bioRxiv preprint doi: https://doi.org/10.1101/2020.1125.398925; this version posted November 26, 2020. The copyright holder for this preprint (which was not certified by peer review) is the author/funder, who has granted bioRxiv a license to display the preprint in perpetuity. It is made available under aCC-BY 4.0 International license.

\title{
Separation and Characterization of Endogenous Nucleosomes by Native Capillary Zone Electrophoresis - Top-Down Mass Spectrometry (nCZE-TDMS)
}

Kevin Jooß ${ }^{\mathrm{a}}$, Luis F. Schachner ${ }^{\mathrm{a}}$, Rachel Watson ${ }^{\mathrm{b}}$, Zachary B. Gillespie ${ }^{\mathrm{b}}$, Sarah A. Howard ${ }^{\mathrm{b}}$, Marcus A. Cheek ${ }^{\mathrm{b}}$, Matthew J. Meiners ${ }^{\mathrm{b}}$, Jonathan D. Licht ${ }^{\mathrm{c}}$, Michael-Christopher Keogh ${ }^{\mathrm{b}}$ \& Neil L. Kelleher ${ }^{\mathrm{a}, *}$

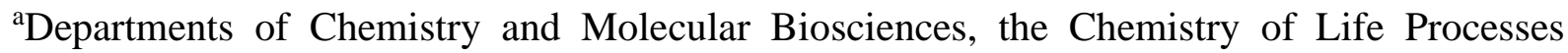
Institute, and the Proteomics Center of Excellence, Northwestern University, Evanston, IL 60208, USA

${ }^{\text {b} E p i C y p h e r ~ I n c ., ~ D u r h a m, ~ N C ~ 27709, ~ U S A ~}$

${ }^{\mathrm{c}}$ Division of Hematology/Oncology, University of Florida Health Cancer Center, Gainesville, FL 32610, USA

*Correspondence: Prof. Dr. Neil Kelleher, 2070 Campus Dr, Evanston, Il 60208, USA, nkelleher@northwestern.edu

\begin{abstract}
Abbreviations: ammonium acetate (AmAc); background electrolyte (BGE); conductive liquid (CL); capillary zone electrophoresis (CZE); designer Nucleosome (dNuc); extracted ion electropherogram (EIE); endogenous nucleosomes (endoNucs); electrospray ionization (ESI); full-width at half maximum (FWHM); acetic acid (HAc), higher-energy collisional dissociation (HCD), hydrochloric acid (HCl); limit of detection (LOD); methylation equivalents (ME); micrococcal nuclease (MNase); molecular weight cut-off (MWCO); native capillary zone electrophoresis (nCZE); native mass spectrometry (nMS), mononucleosome (Nuc), orbitrap (OT); post-translational modification (PTM); Q Exactive HF MS with Extended Mass Range (QE-EMR); recombinant Nucleosome (rNuc); separation line (SL); signal-to-noise ratio (SNR); total ion electropherogram (TIE); top-down mass spectrometry (TDMS); Ultra High Mass Range (UHMR).
\end{abstract}

Keywords: native separation, capillary-zone-electrophoresis mass spectrometry, nucleosome, histone code, native mass spectrometry, native top-down mass spectrometry 


\begin{abstract}
We report a novel platform (native capillary zone electrophoresis - top-down mass spectrometry; nCZE-TDMS) for the separation and characterization of whole nucleosomes, their histone subunits, and PTMs. As the repeating unit of chromatin, mononucleosomes (Nucs) are a $200 \mathrm{kDa}$ complex of DNA and histone proteins involved in the regulation of key cellular processes central to human health and disease. Unraveling the covalent modification landscape of histones and their defined stoichiometries within Nucs helps to explain epigenetic regulatory mechanisms. In nCZE-TDMS, online Nuc separation is followed by a three-tier tandem MS approach that measures the intact mass of Nucs, ejects and detects the constituent histones, and fragments to sequence the histone. The new platform was optimized with synthetic Nucs to reduce both sample requirements and cost significantly compared to direct infusion. Limits of detection were in the low attomole range, with linearity over $\sim$ three orders of magnitude. The nCZE-TDMS platform was applied to endogenous Nucs from two cell lines distinguished by overexpression or knockout of histone methyltransferase NSD2/MMSET, where analysis of constituent histones revealed changes in histone abundances over the course of the CZE separation. We are confident the nCZE-TDMS platform will help advance nucleosome-level research in the fields of chromatin and epigenetics.
\end{abstract}




\section{Introduction}

Increasing evidence indicates that disruption in the cellular epigenetic machinery may be a key initiator in several types of cancer and other disorders. ${ }^{1}$ The mononucleosome (Nuc), the smallest repeating unit of chromatin, resides at the center of epigenetic regulation. It comprises 147 bp of DNA wrapped around a protein octamer with two copies of each core histone: H2A, H2B, H3, and H4 (Figure S1a). ${ }^{2}$ Nucleosome structure (and presumably function) can be altered by covalent modification of the DNA or histone proteins (latter termed post-translational modifications; PTMs), or by replacement of the canonical histones with variants, which have diverse biophysical properties. ${ }^{3,4}$ Nuc modification dynamics can regulate key cellular processes central to human health and disease (e.g. transcription, DNA repair, and DNA replication). ${ }^{5}$ For this reason, the notion of a 'histone code' has gained traction over the last 20 years, given the discovery of effector proteins that simultaneously recognize and bind one or more PTMs on nucleosome assemblies. ${ }^{6}$ Unraveling the full landscape of modifications on whole Nucs is thus key to elucidate the mechanisms that exert epigenetic control over a genomic location.

Chromatin and epigenetics researchers generally use histone peptides as Nuc proxies when studying PTM writers, readers and erasers, obscuring the combinatorial variations that co-occur at the Nuc-level and mediate genome transactions. ${ }^{7,8}$ The high cost of novel PTM-defined Nuc reagents and lack of a direct compositional readout have slowed Nuc-level biology. ${ }^{9,}{ }^{10}$ To address this technology gap, we recently described "Nuc-MS", a novel method based on topdown mass spectrometry (TDMS) operated in native mode, that can decode the protein composition of whole nucleosomes from synthetic and endogenous sources. ${ }^{11}$ A major feature of TDMS is the isolation and fragmentation of individual histones, such that PTMs and variant substitutions can be directly assigned as a single nucleosome proteoform. ${ }^{12}$ The top-down approach has been extended to whole complexes by our group and others ${ }^{13-17}$ and provides a 
higher-level view of the modification landscape compared to the inference required in bottomup approaches. ${ }^{18}$

In recent years, native mass spectrometry (nMS) has greatly contributed to structural biology research. ${ }^{19,20}$ Here, volatile aqueous buffer systems (e.g. ammonium acetate) are employed to preserve near-native features of proteins and their complexes during electrospray ionization. ${ }^{21 \text {, }}$ 22 The approach allows to evaluate the composition of biomolecular structures leading to a better understanding of their biological function and significance. ${ }^{15,22}$ In particular, coupling native ionization with TDMS provides insights to subunit stoichiometry, stability, topology, dynamics, and the affinities of protein complexes. ${ }^{23}$ The lower extent of protonation during native electrospray ionization (ESI) gives access to a wider range of the MS instrument's $\mathrm{m} / \mathrm{z}$ region than under denatured ESI, aiding the resolution of different protein signals. ${ }^{24}$ However, ion suppression and signal superposition cannot be completely avoided for complex samples. Thus, upfront separation under native conditions is beneficial and highly desired.

Different analytical chromatographic $\mathrm{c}^{25-28}$ and electromigrative ${ }^{29-31}$ tools theoretically allow native separation. Generally, there are three major requirements to achieve efficient native separation prior to MS detection: (i) the mobile phase or background electrolyte (BGE) must maintain the nature of the non-denatured protein or complex; (ii) the composition of the mobile phase/BGE must be compatible with electrospray ionization; and (iii) the separation performance and resolution needs to be sufficient. Unfortunately, many current techniques suffer in at least one of these aspects. In contrast, capillary zone electrophoresis (CZE) provides high-resolution even under native conditions. ${ }^{23}$ The separation mechanism of CZE is based on the electrophoretic mobilities of ions in the liquid phase, which are dependent on the chargeto-size ratio of the analytes. ${ }^{32} \mathrm{CZE}$ has been shown to be well-suited for the separation of intact proteins with only small structural differences such as deamidation events. ${ }^{33}$ Another important aspect of CZE is the low sample requirement, typically a few nanoliters per injection. ${ }^{34}$ Several 
CZE-MS interfaces have been recently developed, including sheath liquid, nanoflow sheath liquid and sheathless porous-tip. ${ }^{35}$ However, native (n)CZE-TDMS for protein analysis remains a largely unexplored area, with few studies to date. ${ }^{31,36,37}$

The presence of PTMs and histone variants is generally asserted through immunoblots, affinitybased enrichment (e.g. ChIP-seq), and digestion-based proteomics. ${ }^{38}$ Here, we report the first native-mode MS platform for the separation and characterization of intact Nucs, their histone subunits and PTM profiles in a single experiment. The nCZE-TDMS method was initially optimized using synthetic Nucs to enable $\mathrm{MS}^{1}, \mathrm{MS}^{2}$ and pseudo-MS $\mathrm{M}^{3}$ data collection (Figure S1b), and later expanded for the separation of endogenous nucleosomes (endoNucs) using methyltransferase-modulation cell lines as a model system. An important feature of the platform is that Nuc samples in complex buffers were directly injected into the CZE system without any sample preparation, only consuming minor amounts of sample material $(<\$ 0,01$ per injection). As a result, sample requirements - and thus cost - are significantly reduced compared to direct infusion, enabling the online separation of Nuc sub-populations based on their charge-to-size ratios. This new data type, coupled with Nuc separation by CZE, provides a wide-lens, semiquantitative view of a cell's epigenetic landscape. 


\section{Materials and methods}

\subsection{Materials}

Water $\left(\right.$ Optima $^{\circledR}$ LC-MS grade), acetic acid (HAc) glacial (Optima LC-MS grade), and hydrochloric acid (HCl) (technical grade) were from Thermo Fisher Scientific (Chicago, IL, USA). 7.5 M ammonium acetate (AmAc) stock solution (molecular biology grade) was from Sigma Aldrich (St. Louis, MO, USA). Molecular weight cut-off (MWCO) spin filters were from Thermo Fisher Scientific. All solutions used in the CESI 8000 Plus system were passed through $0.2 \mu \mathrm{m}$ pore size Nalgene ${ }^{\mathrm{TM}}$ Rapid-Flow ${ }^{\mathrm{TM}}$ sterile disposable filters (Thermo Fisher Scientific).

\subsection{Synthetic nucleosome samples}

All semi-synthetic nucleosomes were from EpiCypher (Durham, NC, USA): unmodified recombinant (r)Nuc (16-0006); H3K27me3 (16-0317), H2BK120ub (16-0370) and H3K4,9,14,18ac (16-0336) designer (d)Nucs; and H3.3G34V (16-0347) oncoNuc. For CZE experiments, Nuc samples were kept in their original storage buffer $(10 \mathrm{mM}$ Tris-HCL, $1 \mathrm{mM}$ EDTA, $25 \mathrm{mM} \mathrm{NaCl}, 2 \mathrm{mM}$ DTT and 20\% glycerol) and only mixed and/or diluted in BGE as needed.

For direct infusion experiments via the CESI 8000 Plus device, Nuc samples were buffer exchanged using Amicon Ultra-0.5-mL centrifugal filters (30 kDa MWCO). In brief, the filter device was equilibrated with $500 \mu \mathrm{L}$ of $50 \mathrm{mM}$ AmAc and spun for $5 \mathrm{~min}$ at $12,000 \times \mathrm{g}$. The Nuc sample was then loaded up to a total volume of $500 \mu \mathrm{L}$, and spun for 5 min at $12,000 \times \mathrm{g}$ or until concentrated to $\sim 50 \mu \mathrm{L}$. Eight consecutive washing steps were performed by adding AmAc $(40 \mathrm{mM})$ to $500 \mu \mathrm{L}$ and spinning at $12,000 \times \mathrm{g}$ for $5 \mathrm{~min}$. The remaining sample volume was transferred to CESI NanoVials for analysis. 


\subsection{Endogenous nucleosome samples}

TKO and NTKO NSD2 low and high expressing cells were prepared as described, ${ }^{39}$ with endogenous Nucs extracted and prepared for MS analysis as previously. ${ }^{11}$ The only deviating step was a final buffer exchange to $40 \mathrm{mM}$ AmAc for nCZE-TDMS analysis, resulting in a concentration of about $10 \mathrm{mg} / \mathrm{mL}$ of endoNuc material.

\subsection{Capillary Electrophoresis}

A CESI-8000 Plus instrument from SCIEX (Redwood City, CA, USA) was used, and separation was performed using commercial Neutral OptiMS ${ }^{\mathrm{TM}}$ Capillary Cartridges $(30 \mu \mathrm{m}$ inner diameter, $90 \mathrm{~cm}$ length) containing an integrated sheathless etched porous nanospray tip. The inner wall of the separation capillaries is coated with a binary layer: (i) hydrophobic coating and (ii) hydrophobic polyacrylamide surface. In this way, protein adsorption is prevented, and electro-osmotic flow is largely suppressed.

For initial conditioning, the capillary was rinsed (100 psi) with $0.1 \mathrm{M} \mathrm{HCl} \mathrm{(5} \mathrm{min),} \mathrm{BGE} \mathrm{(10}$ $\mathrm{min}$ ) and water (30 $\mathrm{min})$; the conductive line (CL) rinsed with water (5 min). Subsequently, both separation and conductive line were rinsed (100 psi) with BGE (5 and 3 min, respectively) followed by applying high voltage of $+15 \mathrm{kV}$ and 5 psi supporting flow for $60 \mathrm{~min}$. At the end of this step, the voltage was ramped down over 5 min. Each morning, the capillary was rinsed with fresh BGE and high voltage $(+15 \mathrm{kV}, 5 \mathrm{psi})$ was applied for $30 \mathrm{~min}$. For long term storage, capillaries were rinsed with water and kept at $4{ }^{\circ} \mathrm{C}$.

For Nuc analysis, the capillary was first rinsed with $0.1 \mathrm{M} \mathrm{HCl} \mathrm{(100} \mathrm{psi,} 3 \mathrm{~min})$, followed by BGE (5 min). The CL was filled with 3\% HAc (100 psi, 3 min). Hydrodynamic injection was performed at 2.5 psi for $30 \mathrm{sec}$, corresponding to $\sim 6-38 \mathrm{~nL}(0.9-6.0 \%$ of the total capillary volume), followed by a water dipping step and the injection of a small plug of BGE (2.5 psi, 10 sec). Three different CE methods were developed during this work: (i) standard; (ii) high- 
resolution; and (iii) high-throughput. A detailed description of each individual method is in

\section{Table S1.}

\subsection{Mass Spectrometer}

The CESI-8000 Plus instrument was hyphenated with a custom Thermo Fisher Q Exactive Orbitrap HF MS with Extended Mass Range ${ }^{14}$ (QE-EMR) and a commercial Thermo Fisher Q Exactive Orbitrap MS with Ultra High Mass Range (UHMR). Important parameters of the applied tune files for $\mathrm{MS}^{1}, \mathrm{MS}^{2}$, and pseudo-MS $\mathrm{MS}^{3}$ experiments for both instruments are in Table S2. A Nanospray Flex ${ }^{\mathrm{TM}}$ Ion Source was changed using an OptiMS Thermo MS Adapter from SCIEX for hyphenation of the CESI and orbitrap (OT) instruments. The sprayer tip was positioned $2.5 \mathrm{~mm}$ in front of the OT orifice and an ESI voltage between +1.6 and +1.9 $\mathrm{kV}$ was applied during separation. The inlet capillary of the MS instruments was heated to $330{ }^{\circ} \mathrm{C}(\mathrm{QE}-\mathrm{EMR})$ and $300^{\circ} \mathrm{C}(\mathrm{UHMR})$, respectively.

\subsection{Data Analysis}

Spectra were analyzed manually using Thermo Xcalibur 4.0 Qual Browser (Thermo Fisher Scientific, Inc.). Figures were created using Adobe Illustrator CC 2015.3. Signal-to-Noise ratios (SNR) were calculated as follows: $\mathrm{SNR}=(\mathrm{NL}-\mathrm{B}) /(\mathrm{N}-\mathrm{B})$, where $\mathrm{NL}$ is the signal intensity, $\mathrm{B}$ is the baseline intensity, and $\mathrm{N}$ is the noise intensity. The sum of all observed charge states for each parameter (NL, N and B) was used for SNR calculations. In this way, an average SNR for the protein charge state distribution was determined. Least-square regression was performed, and the residual errors were weighted $1 / \mathrm{x}$, where $\mathrm{x}$ is the respective concentration level. Limit of Detection (LOD, SNR = 3) was estimated by extrapolation of the lowest measured concentration level. Deconvolution of histone data $\left(\mathrm{MS}^{2}\right)$ was performed using Unidec 3.2.0. ${ }^{40}$ Parameters: Data processing: Range 500 - 2500 Th, Bin every: 0; Charge Range: 5 - 15, Mass Range: 10 - 20k Da, Sample mass every (Da): 0.05. Peak area values were 
calculated by integrating the assigned mass range of proteoforms in the deconvoluted mass spectrum. Histone proteoforms were assigned by intact mass and isotopic fit (in-house data base). Statistical significance was evaluated using two-sided, two-sample t-tests and the resulting p-values compared to Bonferroni corrected $\alpha$-values based on $\alpha=0.05 \%$. Mass lists of peptide fragments were created using Xtract (Thermo Fisher Scientific). TDValidator ${ }^{41}$ (max ppm tolerance: 16 ppm; sub ppm tolerance: 7.5 ppm; cluster tolerance: 0.35 ; charge range: 1 15; minimum score: 0.3 ; S/N cutoff: 3 ; Mercury7 Limit: 0.0001 ; minimum size: 2 ) was used to assign recorded fragment ions to the primary sequence of the histone subunits. Electrophoretic resolution was calculated based on the full width half maximum of the respective CZE peaks. 


\section{Results and Discussion}

We first evaluated the performance of the nCZE-TDMS platform with a recombinant Nuc containing the histone $\mathrm{H} 3$ trimethylated at lysine 27 (H3K27me3, $\mathrm{c}=1 \mu \mathrm{M}$, Figure 1a-c). Importantly, the sample was injected directly in the manufacturers storage and shipping buffer (10 mM Tris-HCL, 1 mM EDTA, 25 mM NaCl, 2 mM DTT, 20\% glycerol: EpiCypher) which was designed to minimize Nuc loss and degradation. The data show that both salts and other buffer components are clearly separated from the Nuc signal in the total ion electropherogram (TIE, Figure 1b), obviating the need for additional sample purification such as solvent exchange to AmAc solutions. ${ }^{42}$ A general scheme of the separation system is depicted in Figure 1d. The injection volume was subsequently optimized (Figure S2), and 2.5 psi for $30 \mathrm{~s} \mathrm{(} \mathrm{11}$ nL) selected for further experiments. Comprised of both basic histones ( $\mathrm{pI}>10$ ) and acidic DNA strand $(\mathrm{pI} \approx 5)^{43}$, the effective $\mathrm{pI}$ and mobility of Nucs are hard to predict. Therefore, the separation voltage was varied between +12 and $+18 \mathrm{kV}$ while keeping the remaining method parameters constant to evaluate the migration behavior of Nucs under native conditions (Figure S3). Higher voltages resulted in an increase in migration time, indicating the net charge of the Nuc complexes is negative under the given conditions. Consequently, in the applied positive polarity mode they would counter-migrate towards the CESI inlet, but the supplemental pressure is sufficient to drive the Nucs towards the electrospray source. 

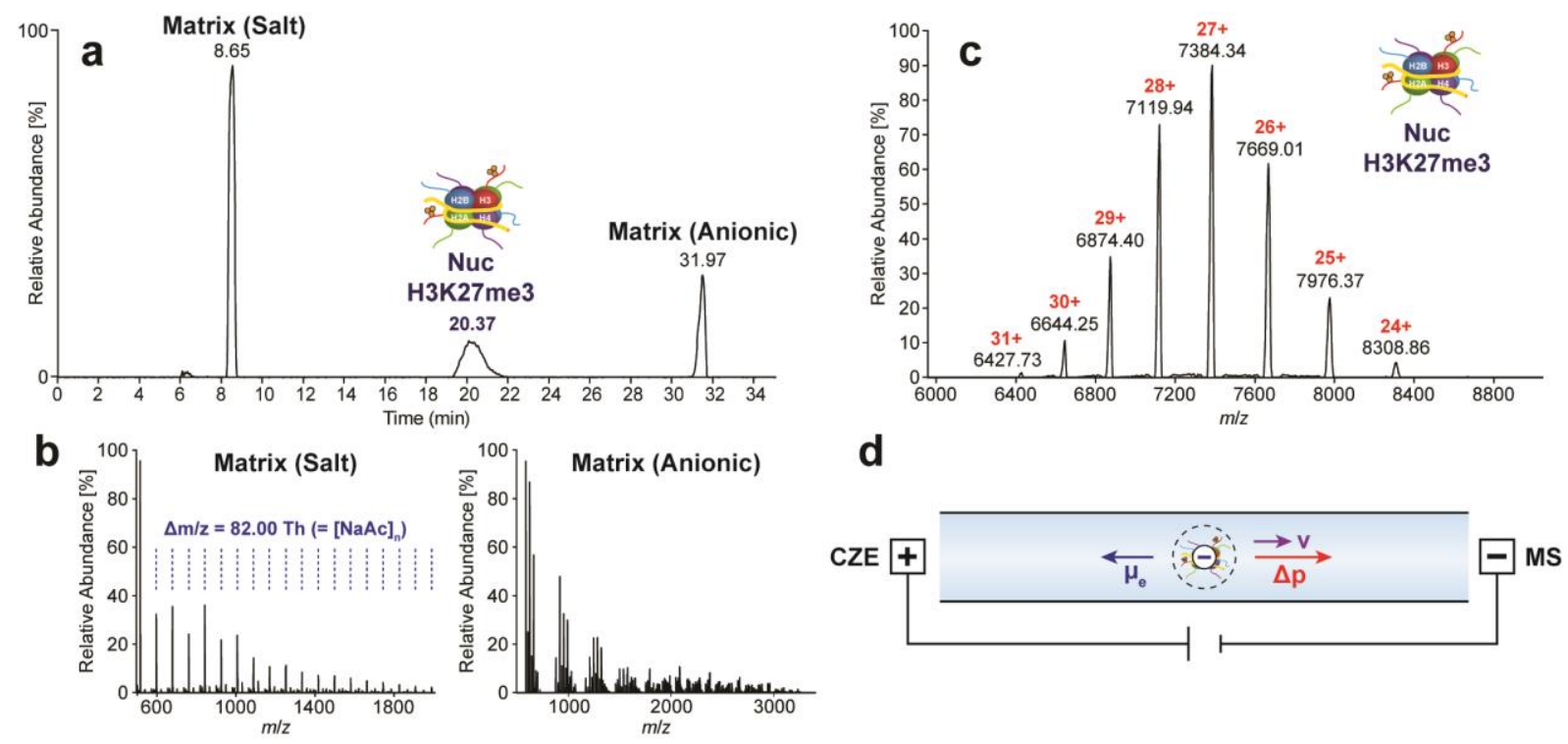

Figure 1: Native CZE-TDMS analysis of Nucs. (a) TIE of Nuc containing H3K27me3 (c = $1 \mu \mathrm{M}$ ) showing the separation of analyte signal and matrix. (b) Mass spectra of high-intensity matrix compounds including sodium acetate clusters. (c) Mass spectrum of intact H3K27me3 nucleosome. (d) Scheme of Nuc migration during analysis. Nucs are negatively charged in AmAc based BGEs $(\mathrm{pH} \approx 6.8)$

We next tested histone ejection from the intact Nucs using this platform. An example $\mathrm{MS}^{2}$ spectrum of H3K27me3 Nucs after ion-source ejection $(c=1 \mu \mathrm{M}$, Figure 2a) demonstrates detection of the four histone subunits $\mathrm{H} 4, \mathrm{H} 2 \mathrm{~B}, \mathrm{H} 2 \mathrm{~A}$, and H3K27me3 (Figure 2b). These ejected histones were subsequently isolated and fragmented by higher-energy collisional dissociation $(\mathrm{HCD})^{14}$ and identified by TDValidator (e.g. H2A: Figure 2c-d). Of note, the quality of fragmentation from online CZE-TDMS was comparable to native direct infusion analyzing the same sample (Figure S4). Another example showing H3K27me3 fragmentation can be found in Figure S5. The native TD approach assigns ejected histones to their related precursor Nuc. Thus, this procedure constitutes an important gain of information relative to denaturing or proteolysis-dependent histone analyses. ${ }^{44}$ 

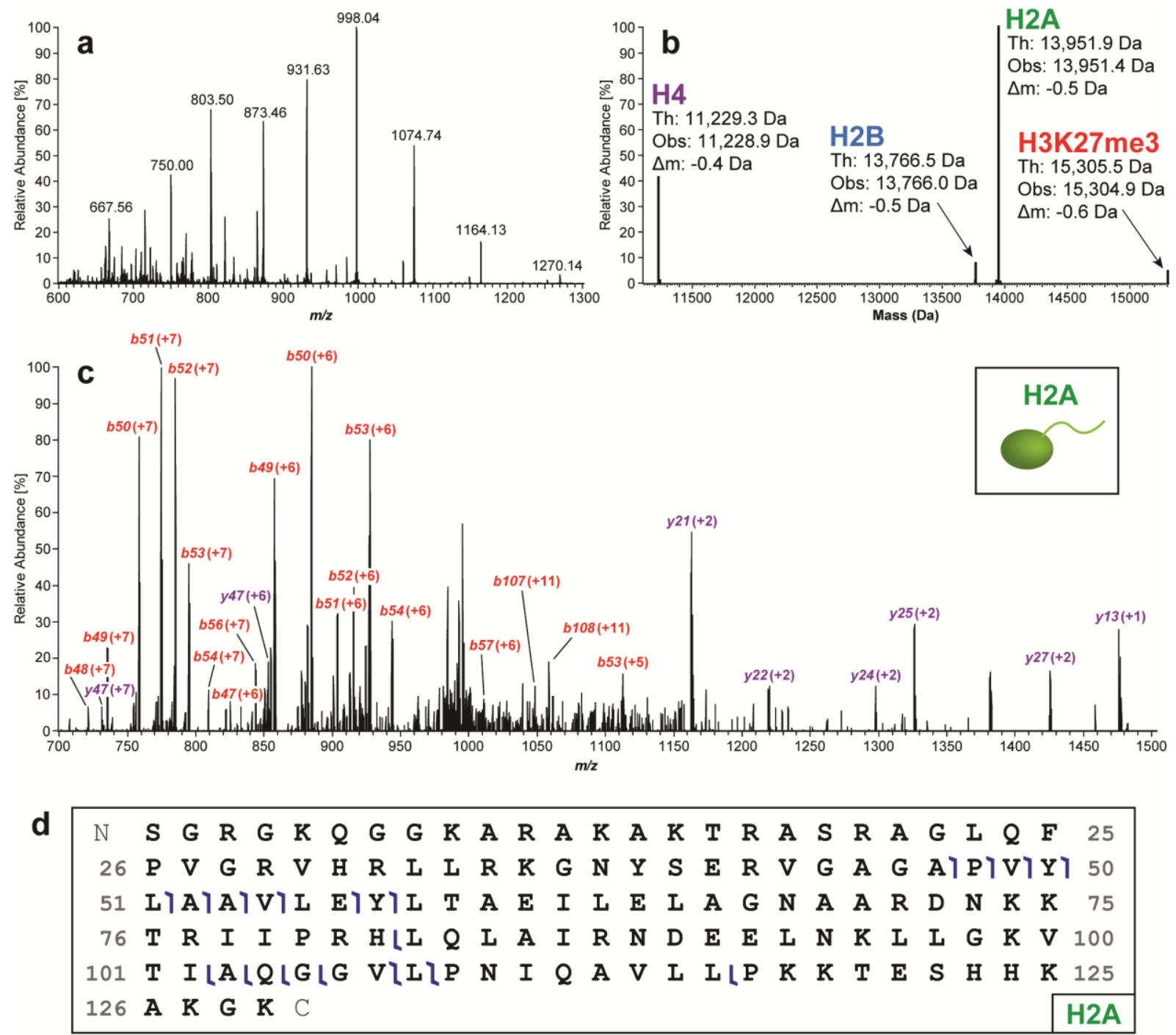

Figure 2: Native CZE-TDMS analysis of H3K27me3 $(c=1 \mu \mathrm{M})$. (a) Mass spectrum of histones ejected from intact Nucs. (b) Deconvoluted mass spectrum of ejected histones including H4, H2B, H2A, and H3K27me3. (c) After histone ejection from the Nuc using in-source dissociation, individual histones were isolated and fragmented by HCD. An example fragment mass spectrum of H2A is depicted. (d) Graphical fragment map of peptides observed from $\mathrm{H} 2 \mathrm{~A}$.

The nCZE-TDMS platform shows high intra- and inter-day reproducibility in migration time, peak area, and intensity using synthetic Nucs, indicating its potential to support the industrial production / analysis of such reagents (Table S3). Moreover, we tested LOD and quantitation at the $\mathrm{MS}^{1}$ level, finding that the linear range of detection is between $10 \mathrm{nM}$ and $500 \mathrm{nM}$

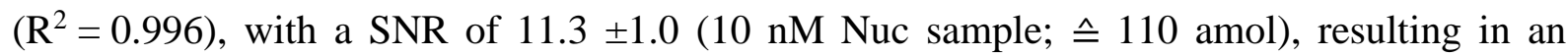


estimated LOD of $2.7 \mathrm{nM}(\bumpeq 30$ amol) (Figure S6a-c). In a follow-up experiment we showed adequate $\mathrm{MS}^{2}$ data for ejected histones from unmodified synthetic Nucs at a concentration of

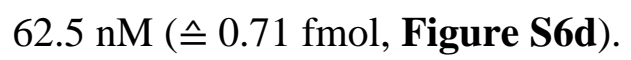

We next sought to investigate the Nuc separation capabilities of the CZE-TDMS platform. For proof-of-concept, we mixed tetra-acetylated (H3K4,9,14,18a) and ubiquitinated (H2BK120ub) Nucs prepared (500 $\mathrm{nM}$ each) as models of highly divergent Nuc species. Tetra-acetylation / charge neutralization of eight lysine residues (i.e. both histone $\mathrm{H} 3$ tails) will decrease the overall net charge of the Nuc complex. The mass shift introduced by two ubiquitins is rather large $(2 \mathrm{x}$ $8.5 \mathrm{kDa}$ ), but it is challenging to predict its influence on the overall net charge of the Nuc complex (the $\sim 6.8 \mathrm{pI}$ of ubiquitin is close to the $\mathrm{pH}$ of the developed CZE method). The mixture was analyzed using the "standard" CZE settings (Figure 3a), achieving partial separation ( $\mathrm{R}=$ 0.366, $\mathrm{n}=2$ ) with $\mathrm{H} 2 \mathrm{BK} 120 \mathrm{ub}$ detected first. Under different parameters (evaluated considering separation performance; Discussion S1) migration times and peak widths increase noticeably when decreasing the supplemental pressure from 5.0 psi to 2.2 psi, electrophoretic resolution is significantly improved from $\mathrm{R}=0.37$ to 1.09 ( $\mathrm{n}=2$ each, Figure $3 \mathbf{b}$ ), which becomes even more evident by comparing the mass spectra in Figure 3c-f. The SNR also improved by three-fold, likely due to reduced ion suppression from overlapping peaks and a higher number of scans averaged. 

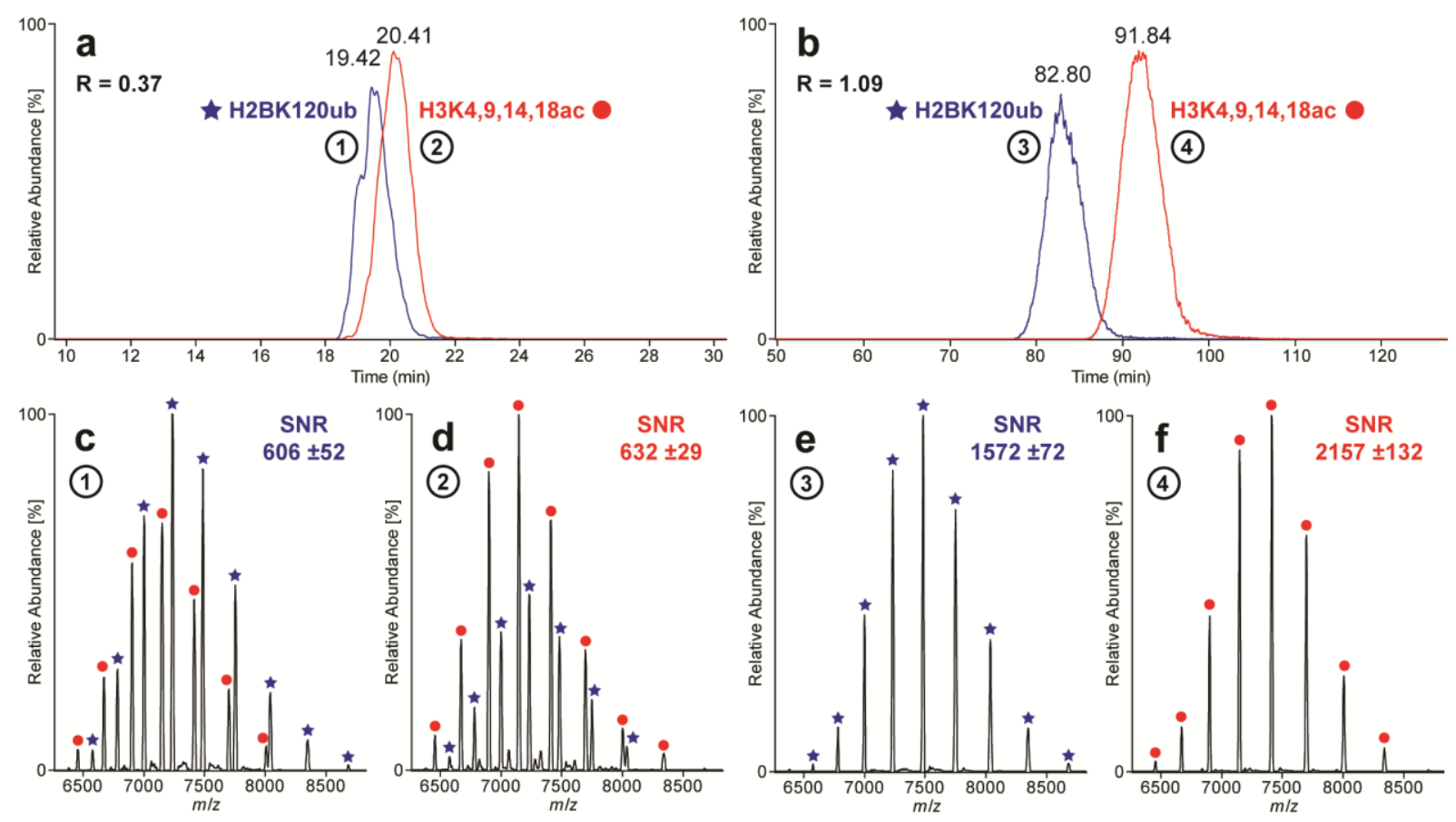

Figure 3: Comparison of "standard" and "high-resolution" CZE methods. Extracted Ion Electropherograms (EIEs, seven highest charge states each) of sample containing H2BK120ub and H3K4,9,14,18ac analyzed with (a) standard and (b) high-resolution CZE method. Mass spectra (c-f) generated by averaging the EIE peaks at full width at half maximum (FWHM). Partial separation $(\mathrm{R}=0.366, \mathrm{n}=2)$ obtained using standard method and close to baseline separation ( $\mathrm{R}=1.082, \mathrm{n}=3)$ achieved for high-resolution method.

In short, the final "high-resolution" CZE method is based on $40 \mathrm{mM} \mathrm{AmAc}(\mathrm{pH}=6.8)$ as BGE and a separation voltage of $+18 \mathrm{kV}$ with 2.2 psi of supplemental pressure, resulting in a total run time of about 135 min. With potential for widespread deployment of the nCZE-TDMS platform, we also developed a "high-throughput" CZE method with a total run time of $\sim 20$ min tailored for the quality control environment during Nuc manufacturing (see Discussion S2).

As a next step, the native CZE-TDMS platform was applied for the analysis of endogenous nucleosomes (endoNucs) derived (see Methods) from an isogenic knockout and overexpression system (respectively TKO and NTKO cell lines ${ }^{45,46}$ ) of NSD2/MMSET, a histone methyltransferase implicated in $\mathrm{t}(4 ; 14)+$ multiple myeloma pathogenesis. In TKO cell lines, the rearranged IGH-NSD2 allele is inactivated by homologous recombination, leaving 
one wild-type copy of the NSD2, which yields low levels of H3K36me2 (NSD2 $^{+/}$; $\mathrm{H} 3 \mathrm{~K} 36 \mathrm{me} 2^{\text {lo }}$ ). In NTKO cells, the wild-type NSD2 allele is inactivated leaving the IGH-NSD2 allele and high levels of H3K36me2 (IGH-NSD2; H3K36me $\left.2^{\text {hi }}\right) .{ }^{45,46}$

The complexity of endoNucs cannot be successfully resolved by traditional direct infusion nMS, which yields a low-abundant and indistinct protein signal (data not shown). In the nCZETDMS system, endoNucs showed increased migration time relative to synthetic Nucs. This indicates a higher electrophoretic mobility, so the supplemental pressure was increased from 2.2 to 3.0 psi (Figure 4a \& Figure S7). The endoNucs migrated over a large time window ( 70 to $80 \mathrm{~min}$ ), which was divided into five-minute subsections and individually deconvoluted to search for distinct populations.

The raw and deconvoluted spectra in Figure $\mathbf{4 b - d}$ reflect a variety of distinct peaks that increase in mass (192,332 to 228,854 Da) over the length of the CZE run. The mass differences between the detected peaks reveals up to 60 "nucleoforms" that differ by a base pair (NTKO: 620.2 $\pm 18.5 \mathrm{Da}$; TKO: $\left.617.5 \pm 22.4 \mathrm{Da} ;\left[\Delta \mathrm{m}_{\mathrm{GC}}=618.4 \mathrm{Da}, \Delta \mathrm{m}_{\mathrm{AT}}=617.4 \mathrm{Da}\right]\right)$; likely reflecting processive micrococcal nuclease (MNase) digestion products. In addition, a series of nonbaseline resolved satellite peaks were observed with a mass shift of $\sim 300 \mathrm{Da}$, indicating single nucleotide differences (Figure $\mathbf{4 b}$ ). These results represent the first report of base pair resolution and accurate mass measurement of intact Nucs from endogenous sources. 

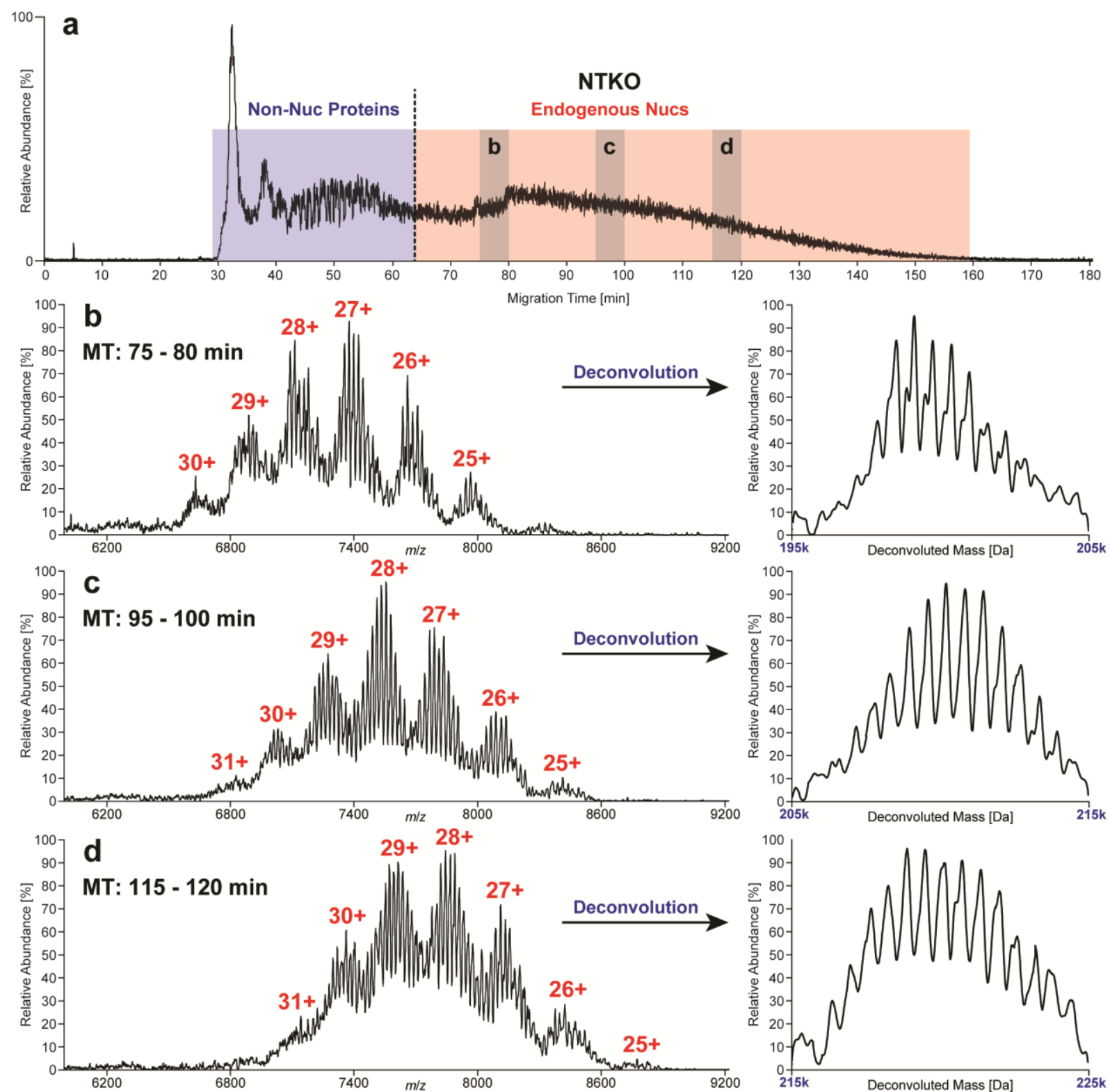

Figure 4: Separation of endoNucs $(\sim 10 \mathrm{mg} / \mathrm{mL})$ derived from NTKO (IGH-NSD2; H3K36me2 ${ }^{\text {hi }}$ ) cells. TIE (a) divided into two major regions: (i) non-Nuc proteins and (ii) endogenous Nucs. Raw and deconvoluted mass spectra (b-d) of three exemplary sections (75 - $80 \mathrm{~min}, 95$ - $100 \mathrm{~min}, 115$ - $120 \mathrm{~min}$ ), where several "nucleoforms" predominated in each. In general, the intact mass of nucleoforms increased with migration time. The average mass shift of $620.2 \pm 18.5$ Da between neighboring peaks, corresponds to a base pair difference in the length of the associated DNA $\left(\Delta \mathrm{m}_{\mathrm{GC}}=618.4 \mathrm{Da}, \Delta \mathrm{m}_{\mathrm{AT}}=617.4 \mathrm{Da}\right)$.

The same samples were analyzed at the histone level $\left(\mathrm{MS}^{2}, \mathrm{n}=3\right.$ per sample), revealing significant proteoform-level differences between NTKO and TKO cells (Figure 5a-d, Table S4). Notably, we detected H3.2 as the predominant H3 subtype in these cells (proteoforms are 
labeled accordingly; Figure S8), although the presence of minor amounts of H3.1 cannot be excluded due to their closely related intact masses. The average methylation equivalent (ME) of H3.2 was decreased by $\sim 11 \%$ in TKO cells, consistent with their reduced expression of NSD2 and lower levels of H3K36 methylation. ${ }^{47}$ Interestingly, the H3.2 4x ME proteoform is highly elevated in TKO, as compared to $6 \mathrm{x}$ and 7x ME in NTKO, suggesting NSD2 cross-talk with pre-existing PTMs. In this regard, H4 acetylation (most likely at K16) ${ }^{11}$ is $21 \%$ elevated in NTKO, suggesting increased areas of active transcription when NSD2 is overexpressed. ${ }^{48}$ Furthermore, the relative abundance of all H3.3 proteoforms is increased in NTKO cells, with $3 x$ to $5 x$ ME readily detected. Finally, the nCZE-TDMS platform directly quantifies $\mathrm{H} 2 \mathrm{~A}$ and H2B variants and their proteoforms (Table S4), revealing modification stoichiometry differences, with potential functional implications. Specifically, there is a higher degree of acetylation on $\mathrm{H} 2 \mathrm{~A} .1 \mathrm{C}$ and $\mathrm{H} 2 \mathrm{~A} .1 \mathrm{~B} / \mathrm{E}$ in NTKO versus TKO cells (17\% and $48 \%$ increase respectively), consistent with the $\mathrm{H} 4$ observation. In terms of $\mathrm{H} 2 \mathrm{~B}$, there is a lower abundance of variants $\mathrm{H} 2 \mathrm{~B} .1-\mathrm{K}$ and $\mathrm{H} 2 \mathrm{~B} .1-\mathrm{C} / \mathrm{E}$ in NTKO relative to TKO cells, suggesting unexplored functional roles for $\mathrm{H} 2 \mathrm{~B}$ variants in epigenetic regulation.

Interestingly, time resolved data shows the relative ratio between histones change significantly over the course of the CZE separation (Figure 5f-g, Table S5 \& S6). In particular, we observed an increase in overall $\mathrm{H} 2 \mathrm{~B}$, with a concomitant decrease in $\mathrm{H} 2 \mathrm{~A}$ and $\mathrm{H} 3$, as the Nuc particles increased in size at higher migration times. This is difficult to explain given our understanding of nucleosome structure and histone stoichiometry / exchange therein. However, inconsistent MNase digestion yields Nucs with varying DNA length (Figure 4), and it is possible that noncovalent interactions between histones and nucleotides in various nucleoforms differentially impacts efficiency of histone ejection (e.g. H2B is less tightly bound, explaining its elevated relative abundance with increasing DNA length). 

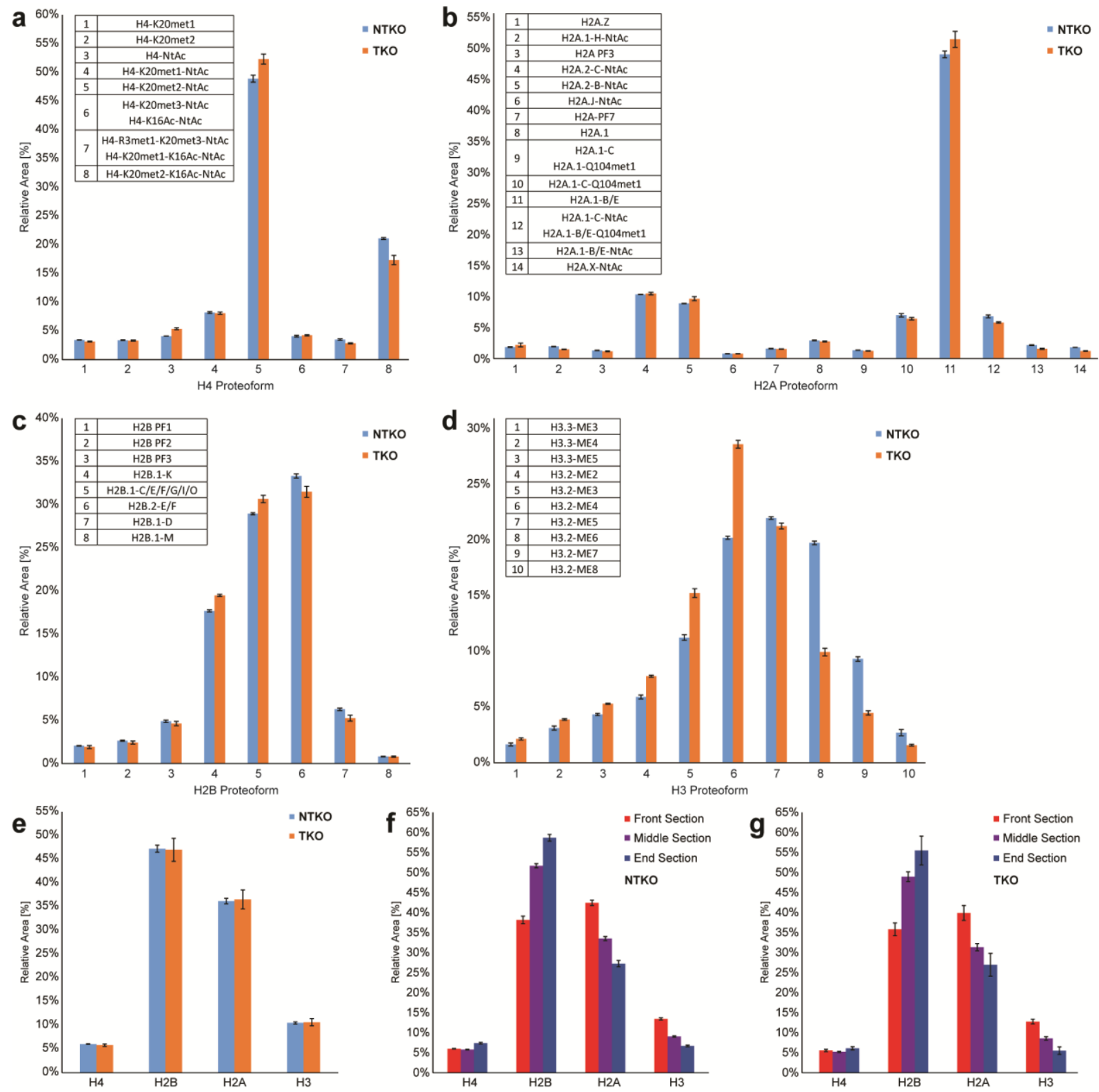

Figure 5: Relative quantification of NTKO/TKO histones using nCZE-TDMS $(n=3)$. (a-d) Histone proteoform level: Averaged mass spectra generated over the entire endoNuc migration range were used and the total peak area of H4 (a), H2A (b), H2B (c), and H3.1 (d) proteoforms was set to 100\%, respectively. (e-g) Histone type level: (e) Comparison between NTKO (IGH-NSD2; H3K36me2 $2^{\text {hi }}$ ) and TKO (NSD2 ${ }^{+-} ;$H3K36me $2^{\text {lo }}$ ) samples using spectra averaged over the entire endoNuc migration time. Comparison between front, middle and end section of the endoNuc migration time window for NTKO (f) and TKO $(\mathrm{g})$. For this purpose, electropherograms were divided into three equally long sections. 
At the proteoform level, some distinct changes over the course of the CZE separation were also observed (Tables S7 \& S8). For example, an increase in H4-K20met2-NtAc $(+14 \%)$ and H2A.1-B/E (+16\%), but decrease in H4-R3met1-K20met3-NtAc / H4-K20met1-K16Ac-NtAc (-42\%), H2A.1 (-34\%), and H2A.1-C-Q104met1 (-30\%) was detected at higher migration times for NTKO (and analogous changes in the proteoforms profiles for TKO). 


\section{Conclusion}

In summary, we have developed the nCZE-TDMS platform to achieve attomole-level Nuc characterization with emphasis on either high resolution or throughput. In this proof-of-concept study, we demonstrate its potential to separate Nucs containing different proteoforms, which has not been shown by other techniques to date. With $\$ 0.01$ of material consumed per 20 min run and minimal sample preparation, the system is a promising candidate for quality control of semi-synthetic Nucs. We challenged the platform with endoNucs from an isogenic methyltransferase knock-out / overexpression cell line system (NTKO/TKO), detecting changes in the $\mathrm{H} 3$ methylation profile consistent with NSD2 status, and proteoforms indicating potential cross-talk with other PTMs, including H4K16ac. Considering CZE separation performance, at the $\mathrm{MS}^{1}$ level we achieved base-pair resolution and detected $\sim 60$ distinct "nucleoforms", associated with differential enzymatic cleavage of DNA by MNase. At the histone level (MS ${ }^{2}$ data) changes in proteoform abundances over the course of nCZE separation were observed for both NTKO and TKO, demonstrating the capabilities to resolve different Nuc sub-populations. We envision extending into the area of endogenous Nucs from cells and immuno-precipitated specimens where limiting amounts of starting material are available. In future studies, we aspire to simplify the complexity of nucleoforms by optimizing the digestion process to get a better overview of proteoforms changes. In conclusion, nCZE-TDMS has the potential to serve as an important tool in the field of epigenetics, native proteomics ${ }^{17}$ and in the quality control of complex synthetic biomolecules. 


\section{Acknowledgements}

This work was supported by the National Institute of General Medical Sciences P41 GM108569

for the National Resource for Translational and Developmental Proteomics at Northwestern University and NIH grants S10 OD025194 and RF1 AG063903 (Kelleher lab), R01 CA195732 and a Leukemia and Lymphoma Society Specialized Center of Excellence Grant (Licht Lab) and R44 GM116584 and R44 CA212733 (EpiCypher). LFS is a Gilliam Fellow of the Howard Hughes Medical Institute. Research in this publication is also supported by Thermo Fisher Scientific and a fellowship associated with the Chemistry of Life Processes Predoctoral Training Grant T32 GM105538 at Northwestern University. We thank SCIEX for their support including Dr. Fang Wang for the valuable discussions and insightful suggestions throughout this research project.

\section{Conflicts of interest}

NLK serves as a consultant to Thermo Fisher Scientific and engages in entrepreneurship in the area of top-down proteomics. EpiCypher is a commercial developer and supplier of the semisynthetic modified nucleosomes (dNucs) used in this study. 


\section{References}

1. Portela, A.; Esteller, M., Epigenetic modifications and human disease. Nat Biotechnol 2010, 28 (10), 1057-1068.

2. Richmond, T. J., Hot papers - Crystal structure - Crystal structure of the nucleosome core particle at 2.8 angstrom resolution by K. Luger, A.W. Mader, R.K. Richmond, D.F. Sargent, T.J. Richmond - Comments. Scientist 1999, 13 (23), 15-15.

3. Patel, D. J.; Wang, Z. X., Readout of Epigenetic Modifications. Annu Rev Biochem 2013, 82, 81-+.

4. Zink, L. M.; Hake, S. B., Histone variants: nuclear function and disease. Curr Opin Genet Dev 2016, 37, 82-89.

5. Bannister, A. J.; Kouzarides, T., Regulation of chromatin by histone modifications. Cell Res 2011, 21 (3), 381-395.

6. Jenuwein, T.; Allis, C. D., Translating the histone code. Science 2001, 293 (5532), 1074-1080.

7. Strelow, J. M.; Xiao, M.; Cavitt, R. N.; Fite, N. C.; Margolis, B. J.; Park, K. J., The Use of Nucleosome Substrates Improves Binding of SAM Analogs to SETD8. J Biomol Screen 2016, 21 (8), 786-794.

8. $\quad$ Connelly, K. E.; Weaver, T. M.; Alpsoy, A.; Gu, B. X.; Musselman, C. A.; Dykhuizen, E. C., Engagement of DNA and H3K27me3 by the CBX8 chromodomain drives chromatin association. Nucleic Acids Res 2019, 47 (5), 2289-2305.

9. Muller, M. M.; Muir, T. W., Histones: At the Crossroads of Peptide and Protein Chemistry. Chem Rev 2015, 115 (6), 2296-2349.

10. Nguyen, U. T. T.; Bittoval, L.; Muller, M. M.; Fierz, B.; David, Y.; Houck-Loomis, B.; Feng, V.; Dann, G. P.; Muir, T. W., Accelerated chromatin biochemistry using DNAbarcoded nucteosome libraries. Nature Methods 2014, 11 (8), 834-840.

11. Schachner, L. F.; Jooß, K.; Morgan, M. A.; Piunti, A.; Meiners, M. J.; Lee, A.; Kafader, J. O.; Iwanaszko, M.; Cheek, M. A.; Burg, J. M.; Howard, S. A.; Keogh, M.-C.; Shilatifard, A.; Kelleher, N. L., Decoding the Protein Composition of Whole Nucleosomes with Nuc-MS. bioRxiv 2020, 2020.09.08.287656.

12. Smith, L. M.; Kelleher, N. L.; Consortium for Top Down, P., Proteoform: a single term describing protein complexity. Nat Methods 2013, 10 (3), 186-7.

13. Schachner, L. F.; Ives, A. N.; McGee, J. P.; Melani, R. D.; Kafader, J. O.; Compton, P. D.; Patrie, S. M.; Kelleher, N. L., Standard Proteoforms and Their Complexes for Native Mass Spectrometry. J Am Soc Mass Spectr 2019, 30 (7), 1190-1198.

14. Belov, M. E.; Damoc, E.; Denisov, E.; Compton, P. D.; Horning, S.; Makarov, A. A.; Kelleher, N. L., From Protein Complexes to Subunit Backbone Fragments: A Multi-stage Approach to Native Mass Spectrometry. Anal Chem 2013, 85 (23), 11163-11173.

15. Li, H. L.; Nguyen, H. H.; Loo, R. R. O.; Campuzano, I. D. G.; Loo, J. A., An integrated native mass spectrometry and top-down proteomics method that connects sequence to structure and function of macromolecular complexes. Nat Chem 2018, 10 (2), 139-148.

16. Zhang, H.; Cui, W. D.; Wen, J. Z.; Blankenship, R. E.; Gross, M. L., Native Electrospray and Electron-Capture Dissociation in FTICR Mass Spectrometry Provide TopDown Sequencing of a Protein Component in an Intact Protein Assembly. J Am Soc Mass Spectr 2010, 21 (12), 1966-1968.

17. Skinner, O. S.; Haverland, N. A.; Fornelli, L.; Melani, R. D.; Do Vale, L. H. F.; Seckler, H. S.; Doubleday, P. F.; Schachner, L. F.; Srzentic, K.; Kelleher, N. L.; Compton, P. D., Top-down characterization of endogenous protein complexes with native proteomics. Nat Chem Biol 2018, 14 (1), 36-+. 
18. Kelleher, N. L.; Lin, H. Y.; Valaskovic, G. A.; Aaserud, D. J.; Fridriksson, E. K.; McLafferty, F. W., Top down versus bottom up protein characterization by tandem highresolution mass spectrometry. J Am Chem Soc 1999, 121 (4), 806-812.

19. Heck, A. J.; Van Den Heuvel, R. H., Investigation of intact protein complexes by mass spectrometry. Mass Spectrom Rev 2004, 23 (5), 368-89.

20. Hilton, G. R.; Benesch, J. L., Two decades of studying non-covalent biomolecular assemblies by means of electrospray ionization mass spectrometry. $J R$ Soc Interface 2012, 9 (70), 801-16.

21. de la Mora, J. F., Electrospray ionization of large multiply charged species proceeds via Dole's charged residue mechanism. Anal Chim Acta 2000, 406 (1), 93-104.

22. Leney, A. C.; Heck, A. J. R., Native Mass Spectrometry: What is in the Name? J Am Soc Mass Spectr 2017, 28 (1), 5-13.

23. Belov, A. M.; Viner, R.; Santos, M. R.; Horn, D. M.; Bern, M.; Karger, B. L.; Ivanov, A. R., Analysis of Proteins, Protein Complexes, and Organellar Proteomes Using Sheathless Capillary Zone Electrophoresis - Native Mass Spectrometry. J Am Soc Mass Spectr 2017, 28 (12), 2614-2634.

24. Kafader, J. O.; Melani, R. D.; Schachner, L. F.; Ives, A. N.; Patrie, S. M.; Kelleher, N. L.; Compton, P. D., Native vs Denatured: An in Depth Investigation of Charge State and Isotope Distributions. J Am Soc Mass Spectr 2020, 31 (3), 574-581.

25. Debaene, F.; Boeuf, A.; Wagner-Rousset, E.; Colas, O.; Ayoub, D.; Corvaia, N.; Van Dorsselaer, A.; Beck, A.; Cianferani, S., Innovative native MS methodologies for antibody drug conjugate characterization: High resolution native MS and IM-MS for average DAR and DAR distribution assessment. Anal Chem 2014, 86 (21), 10674-83.

26. Muneeruddin, K.; Nazzaro, M.; Kaltashov, I. A., Characterization of Intact Protein Conjugates and Biopharmaceuticals Using Ion-Exchange Chromatography with Online Detection by Native Electrospray Ionization Mass Spectrometry and Top-Down Tandem Mass Spectrometry. Anal Chem 2015, 87 (19), 10138-10145.

27. Gahoual, R.; Heidenreich, A. K.; Somsen, G. W.; Bulau, P.; Reusch, D.; Wuhrer, M.; Haberger, M., Detailed Characterization of Monoclonal Antibody Receptor Interaction Using Affinity Liquid Chromatography Hyphenated to Native Mass Spectrometry. Anal Chem 2017, 89 (10), 5404-5412.

28. Kukrer, B.; Filipe, V.; van Duijn, E.; Kasper, P. T.; Vreeken, R. J.; Heck, A. J.; Jiskoot, W., Mass spectrometric analysis of intact human monoclonal antibody aggregates fractionated by size-exclusion chromatography. Pharm Res 2010, 27 (10), 2197-204.

29. Fonslow, B. R.; Kang, S. A.; Gestaut, D. R.; Graczyk, B.; Davis, T. N.; Sabatini, D. M.; Yates, J. R., 3rd, Native capillary isoelectric focusing for the separation of protein complex isoforms and subcomplexes. Anal Chem 2010, 82 (15), 6643-51.

30. Reschiglian, P.; Zattoni, A.; Roda, B.; Roda, A.; Parisi, D.; Moon, M. H.; Min, B. R., Hollow-fiber flow field-flow fractionation: a gentle separation method for mass spectrometry of native proteins. Ann Chim 2006, 96 (5-6), 253-7.

31. Gomes, F. P.; Yates, J. R., 3rd, Recent trends of capillary electrophoresis-mass spectrometry in proteomics research. Mass Spectrom Rev 2019.

32. Jorgenson, J. W.; Lukacs, K. D., Zone Electrophoresis in Open-Tubular GlassCapillaries. Anal Chem 1981, 53 (8), 1298-1302.

33. Jooss, K.; Huhner, J.; Kiessig, S.; Moritz, B.; Neususs, C., Two-dimensional capillary zone electrophoresis-mass spectrometry for the characterization of intact monoclonal antibody charge variants, including deamidation products. Anal Bioanal Chem 2017, 409 (26), 6057-6067.

34. Issaq, H. J.; Janini, G. M.; Atamna, I. Z.; Muschik, G. M., Separations by HighPerformance Liquid-Chromatography and Capillary Zone Electrophoresis - a ComparativeStudy. J Liq Chromatogr 1991, 14 (5), 817-845. 
bioRxiv preprint doi: https://doi.org/10.1101/2020.11.25.398925; this version posted November 26, 2020. The copyright holder for this preprint (which was not certified by peer review) is the author/funder, who has granted bioRxiv a license to display the preprint in perpetuity. It is made available under aCC-BY 4.0 International license.

35. Hocker, O.; Montealegre, C.; Neususs, C., Characterization of a nanoflow sheath liquid interface and comparison to a sheath liquid and a sheathless porous-tip interface for CE-ESI-MS in positive and negative ionization. Anal Bioanal Chem 2018, 410 (21), 52655275.

36. Shen, X. J.; Yang, Z. C.; McCool, E. N.; Lubeckyj, R. A.; Chen, D. Y.; Sun, L. L., Capillary zone electrophoresis-mass spectrometry for top-down proteomics. Trac-Trend Anal Chem 2019, 120.

37. Mehaffey, M. R.; Xia, Q.; Brodbelt, J. S., Uniting Native Capillary Electrophoresis and Multistage Ultraviolet Photodissociation Mass Spectrometry for Online Separation and Characterization of Escherichia coli Ribosomal Proteins and Protein Complexes. Anal Chem 2020, 92 (22), 15202-15211.

38. Moller, J.; de Pablo, J. J., Bottom-Up Meets Top-Down: The Crossroads of Multiscale Chromatin Modeling. Biophys J 2020, 118 (9), 2057-2065.

39. Lauring, J.; Abukhdeir, A. M.; Konishi, H.; Garay, J. P.; Gustin, J. P.; Wang, Q.; Arceci, R. J.; Matsui, W.; Park, B. H., The multiple myeloma-associated MMSET gene contributes to cellular adhesion, clonogenic growth, and tumorigenicity. Blood 2008, 111 (2), 856-864.

40. Marty, M. T.; Baldwin, A. J.; Marklund, E. G.; Hochberg, G. K. A.; Benesch, J. L. P.; Robinson, C. V., Bayesian Deconvolution of Mass and Ion Mobility Spectra: From Binary Interactions to Polydisperse Ensembles. Anal Chem 2015, 87 (8), 4370-4376.

41. Fornelli, L.; Srzentic, K.; Huguet, R.; Mullen, C.; Sharma, S.; Zabrouskoy, V.; Fellers, R. T.; Durbin, K. R.; Compton, P. D.; Kelleher, N. L., Accurate Sequence Analysis of a Monoclonal Antibody by Top-Down and Middle-Down Orbitrap Mass Spectrometry Applying Multiple Ion Activation Techniques. Anal Chem 2018, 90 (14), 8421-8429.

42. Susa, A. C.; Xia, Z. J.; Williams, E. R., Small Emitter Tips for Native Mass Spectrometry of Proteins and Protein Complexes from Nonvolatile Buffers That Mimic the Intracellular Environment. Anal Chem 2017, 89 (5), 3116-3122.

43. Marino-Ramirez, L.; Kann, M. G.; Shoemaker, B. A.; Landsman, D., Histone structure and nucleosome stability. Expert Rev Proteomic 2005, 2 (5), 719-729.

44. Zheng, Y. P.; Huang, X. X.; Kelleher, N. L., Epiproteomics: quantitative analysis of histone marks and codes by mass spectrometry. Curr Opin Chem Biol 2016, 33, 142-150.

45. Popovic, R.; Martine-Garcia, E.; Giannopoulou, E. G.; Zhang, Q. W.; Zhang, Q. Y.; Ezponda, T.; Shah, M. Y.; Zheng, Y. P.; Will, C. M.; Small, E. C.; Hua, Y. J.; Bulic, M.; Jiang, Y. W.; Carrara, M.; Calogero, R. A.; Kath, W. L.; Kelleher, N. L.; Wang, J. P.; Elemento, O.; Licht, J. D., Histone Methyltransferase MMSET/NSD2 Alters EZH2 Binding and Reprograms the Myeloma Epigenome through Global and Focal Changes in H3K36 and H3K27 Methylation. Plos Genet 2014, 10 (9).

46. Zheng, Y. P.; Sweet, S. M. M.; Popovic, R.; Martinez-Garcia, E.; Tipton, J. D.; Thomas, P. M.; Licht, J. D.; Kelleher, N. L., Total kinetic analysis reveals how combinatorial methylation patterns are established on lysines 27 and 36 of histone H3. P Natl Acad Sci USA 2012, 109 (34), 13549-13554.

47. Martinez-Garcia, E.; Popovic, R.; Min, D. J.; Sweet, S. M. M.; Thomas, P. M.; Zamdborg, L.; Heffner, A.; Will, C.; Lamy, L.; Staudt, L. M.; Levens, D. L.; Kelleher, N. L.; Licht, J. D., The MMSET histone methyl transferase switches global histone methylation and alters gene expression in t(4;14) multiple myeloma cells. Blood 2011, 117 (1), 211-220. 48. Taylor, G. C. A.; Eskeland, R.; Hekimoglu-Balkan, B.; Pradeepa, M. M.; Bickmore, W. A., H4K16 acetylation marks active genes and enhancers of embryonic stem cells, but does not alter chromatin compaction. Genome Res 2013, 23 (12), 2053-2065. 